\title{
LA-UR-20-28486
}

Approved for public release; distribution is unlimited.

Title:

Author(s):

Intended for:

Issued:
LANSCE Module 1 Capacitor Room Failure

Taylor, Antoinette Jane

Report

$2020-10-21$ 
Disclaimer:

Los Alamos National Laboratory, an affirmative action/equal opportunity employer, is operated by Triad National Security, LLC for the National Nuclear Security Administration of U.S. Department of Energy under contract 89233218CNA000001. By approving this article, the publisher recognizes that the U.S. Government retains nonexclusive, royalty-free license to publish or reproduce the published form of this contribution, or to allow others to do so, for U.S. Government purposes. Los Alamos National Laboratory requests that the publisher identify this article as work performed under the auspices of the U.S. Department of Energy. Los Alamos National Laboratory strongly supports academic freedom and a researcher's right to publish; as an institution, however, the Laboratory does not endorse the viewpoint of a publication or guarantee its technical correctness. 


\section{LANSCE Module 1 Capacitor Room Failure}

ALDPS - AOT Division When things go right

Toni Taylor

October 8, 2020

- Los Alamos NATIONAL LABORATORY 


\section{To achieve Simultaneous Excellence, a strong safety culture}

is required...

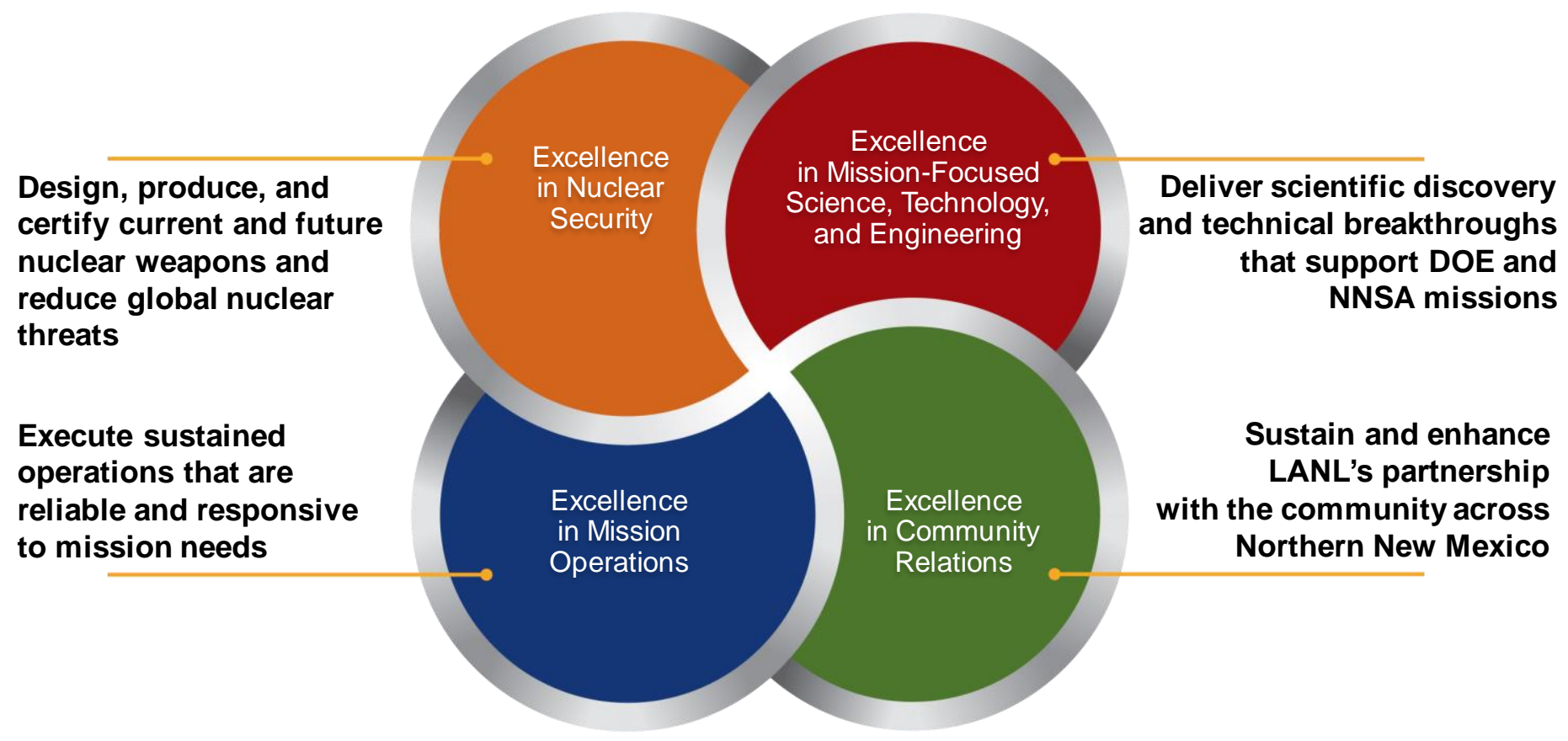

How we do our work is as important as what we do 


\section{The how is defined by our behaviors}

- Collaborative Problem Solving

- Demonstrate initiative and wiliness to work with others and be inclusive

- Continuous Learning

- Proactively develop, adapt and transform oneself and shares lessons learned; apply feedback and lessons learned.

\section{- Shared Outcome}

- Demonstrate a shared purpose within the Lab. Respect others and maintain a positive/responsive attitude. Encourages and supports security and safety.

- Trustworthy

- Communicate openly, honestly, and with integrity. Demonstrates follow through.

- Commitment

- Stay motivated, focused and dedication to a position or plan of action. 


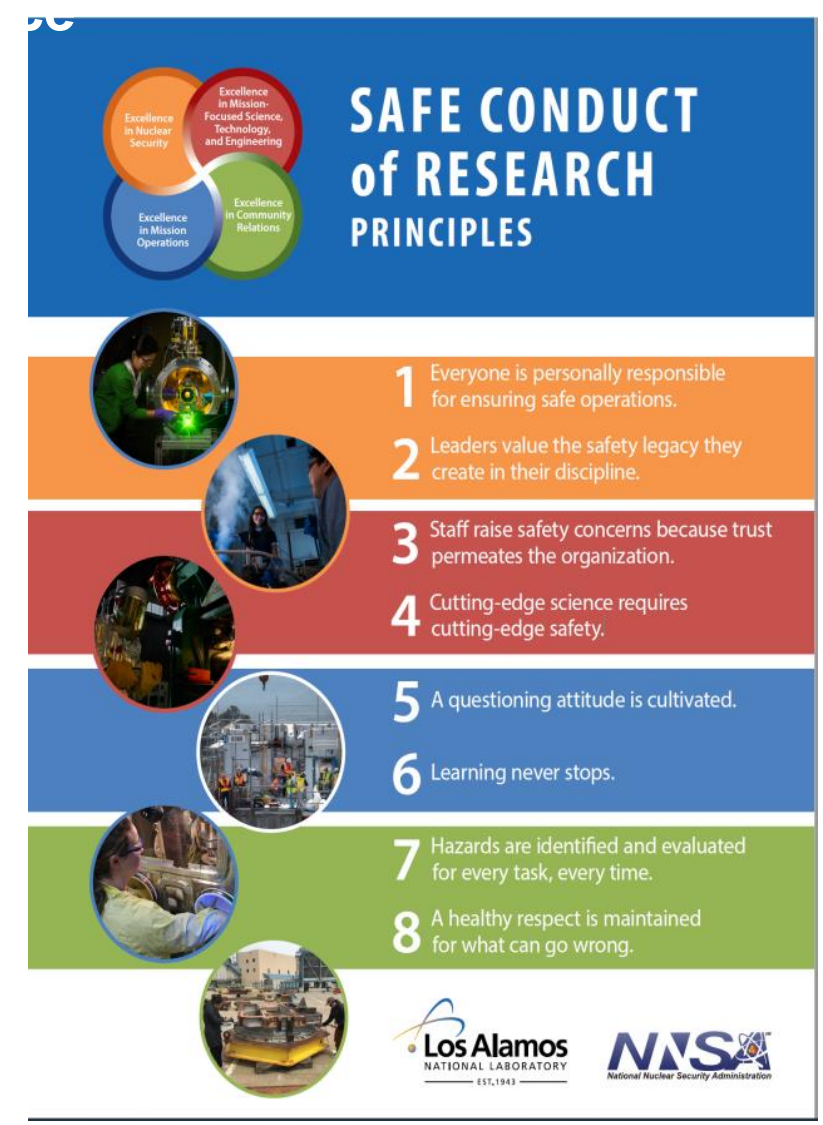

These principles help us all have a shared understanding of the need to plan, execute, and evaluate critically. They reinforce the need to:

- Stop when unsure

- Protect yourself

- Be your co-worker's keeper 


\section{Summary of Event}

- At 3:30 am on Sunday, high-voltage line in capacitor room overheated to the extent that the poly insulating material caught fire and dripped melted insulation onto equipment below.

- LAFD responded and, per pre-incident planning, waited for AOT personnel to respond to the area.

- AOT personnel, safed equipment and waited with LAFD for fire to "burn out".

- Using an IWD, AOT personnel safely re-entered the area after conducting hazards analysis and engaging SMEs re: controls for combustion products and COVID-19 hazards.

- Recovery action took several days with a focus on getting it right

- Beam re-start decisions were made with impacted organizations and considering AOTRFE personnel

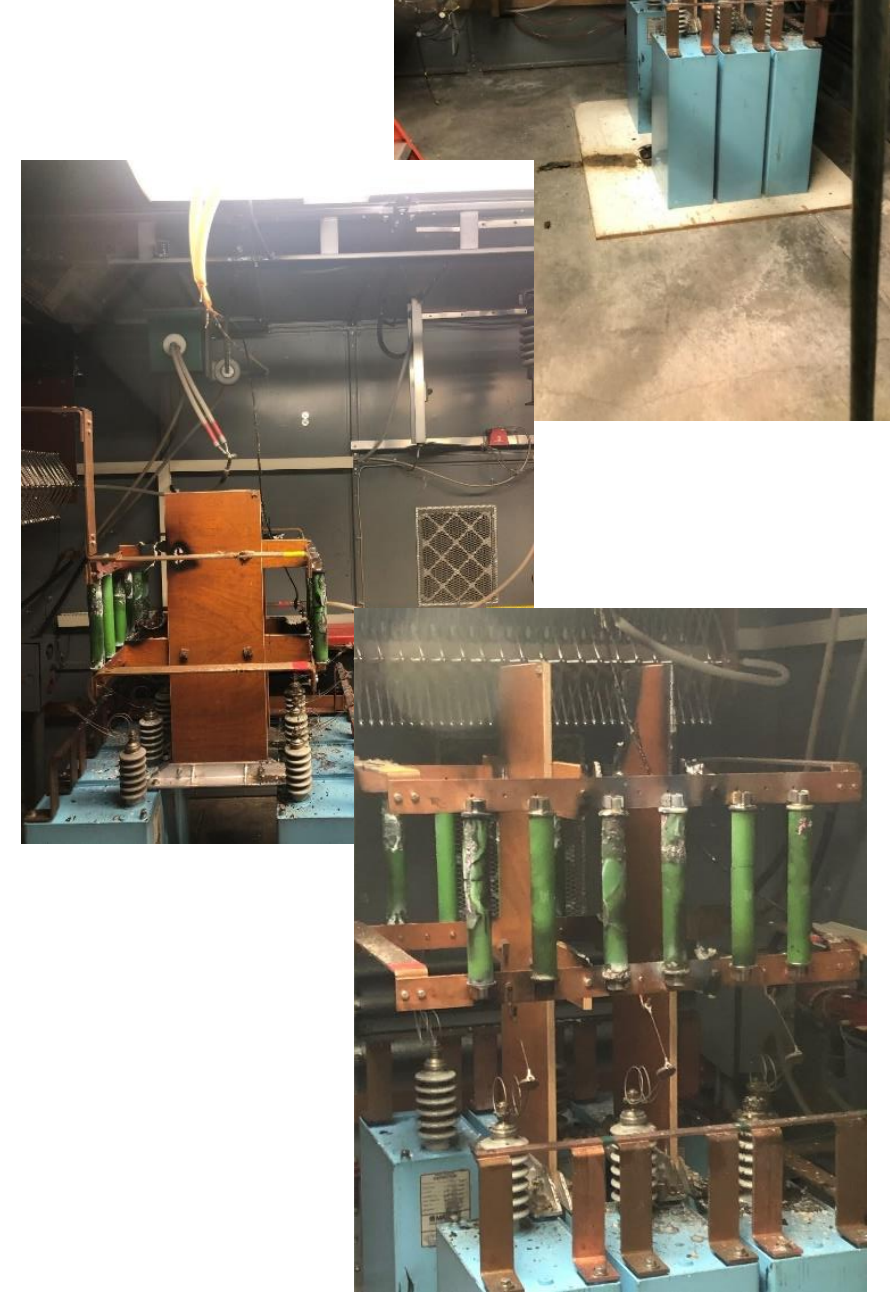

Beam was restored at reduced capacity yesterday and full capacity four days post event; if necessary, repairs will occur in November 


\section{Response and Recovery Actions}

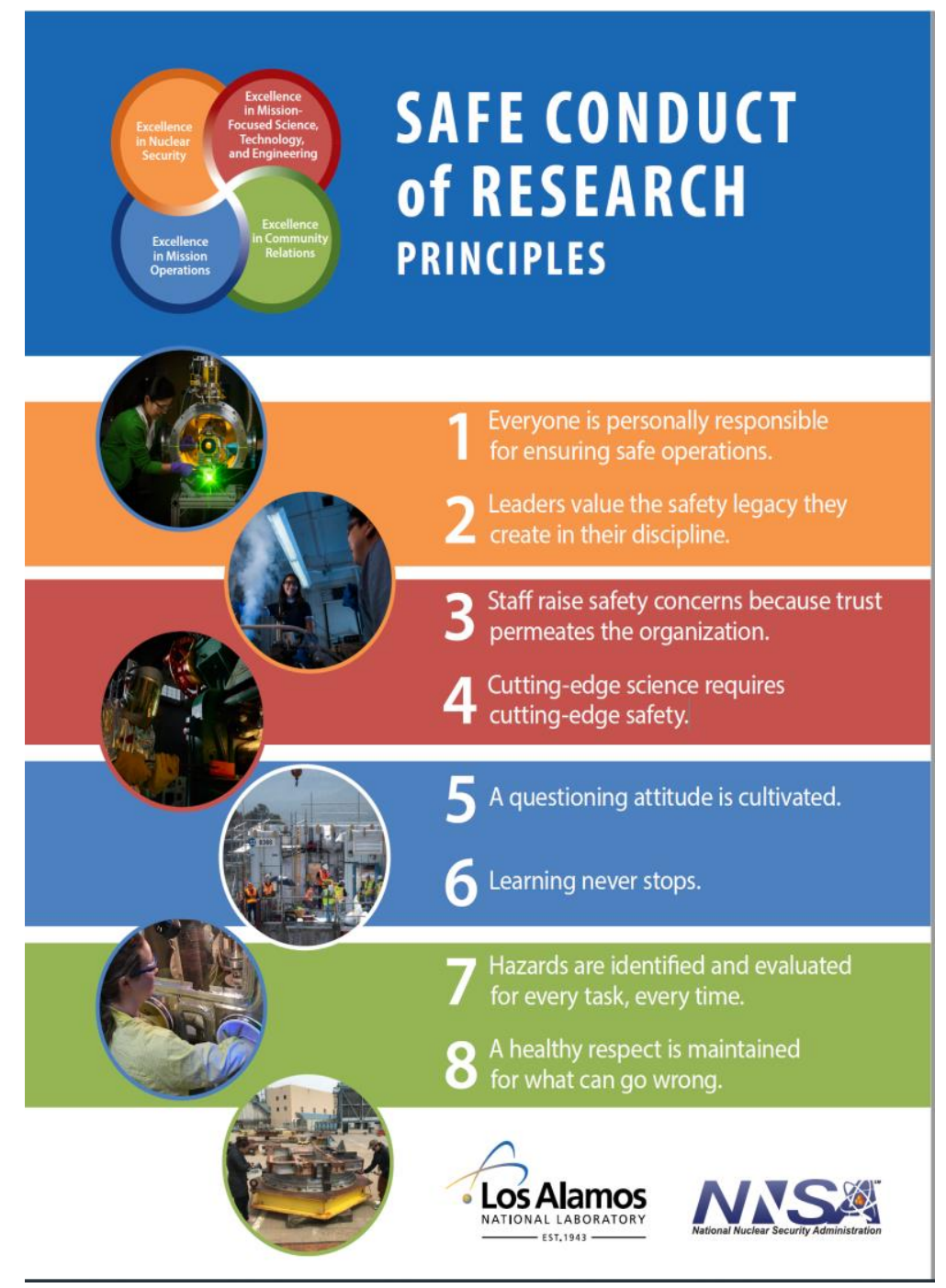

- SMEs and RLMs were engaged

- Safety of personnel, understanding what occurred to prevent recurrence were priorities

- Hazards analysis and controls verification were executed prior to reentry

- Extent of condition for other cap banks evaluated prior to re-start

- Designated stop times were identified,

- Not unanticipated failure

- Pre-incident planning paid off - LAFD waited for AOT personnel 


\section{Backup}

